
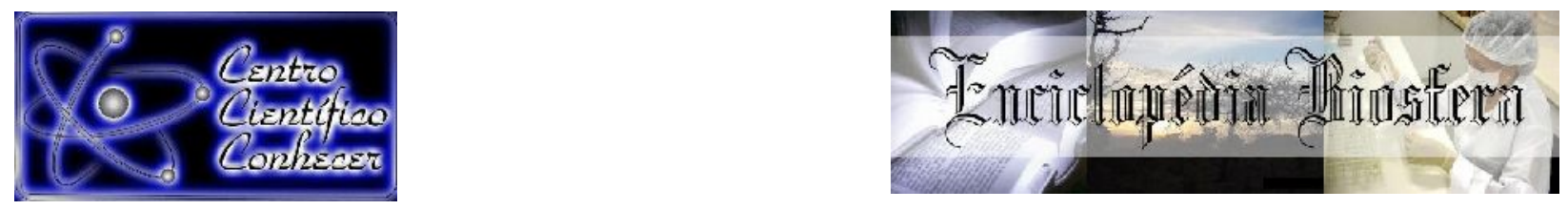

\title{
BRIÓFITAS DE UMA ÁREA ECOTONAL DE CAATINGA/CERRADO NO ESTADO DO PIAUÍ, BRASIL
}

\author{
Maria Elizabeth Barbosa da Sousa ${ }^{1}$, Hermeson Cassiano de Oliveira ${ }^{1}$ \\ ${ }^{1}$ Universidade Estadual do Piauí, Campus Heróis do Jenipapo, Herbário de \\ Criptógamas, Av. Sto. Antônio s/n, 64280-000 Campo Maior, PI, Brasil. E-mail: \\ ellizabethsousa@gmail.com
}

Recebido em: 06/04/2019 - Aprovado em: 10/06/2019 - Publicado em: 30/06/2019 DOI: 10.18677/EnciBio_2019A138

\begin{abstract}
RESUMO
O estado do Piauí caracteriza por apresentar extensas áreas de tensão ecológica, com vegetação de transição entre caatinga e manchas de cerrado caducifólio. Em relação à sua flora de briófitas, apenas 49 espécies estão registradas para o estado. O objetivo do trabalho foi conhecer a diversidade de musgos e hepáticas ocorrente em uma área de transição entre Caatinga e Cerrado no estado do Piauí, visando ampliar o conhecimento sobre essas plantas no estado. Foram encontradas 39 espécies de briófitas e uma variedade, distribuídas em 13 famílias e 22 gêneros, das quais 22 são novas ocorrências para o estado do Piauí, 17 para a Caatinga e duas para o Cerrado. A divisão Bryophyta apresentou 25 espécies, enquanto a divisão Marchantiophyta resultou em 14 espécies. A Família Fissidentaceae foi a mais representativa entre os musgos, com oito espécies, ja para a hepáticas, a família mais abundante foi Lejeuneaceae, com 10 espécies. Quanto aos substratos colonizados, predominou-se o rupícolo, seguido de corticícolo, terrícolo e epíxilo. O inventário florístico possibilitou ampliar a distribuição geográfica das espécies para o estado do Piauí e entre os domínios fitogeográficos do Brasil, fornecendo novos registros para Caatinga e Cerrado.
\end{abstract}

PALAVRAS-CHAVE: florística, hepáticas, musgos.

\section{BRYOPHYTES OF AN ECOTONAL AREA BETWEEN CAATINGA / CERRADO IN THE STATE OF PIAUÍ, BRAZIL}

\begin{abstract}
The state of Piauí is characterized by presenting large areas of ecological tension, with a transition vegetation between Caatinga and Cerrado. In relation to the bryophyte flora, only 49 species are recorded for the state. We aim to know the diversity of mosses and liverworts occur in an area of transition between Caatinga and Cerrado in the state of Piauí, in order to extend the knowledge about these plants in the state. We found 39 species of bryophytes and one variety, distribuited in 13 families and 22 genera, of which 22 are new occurrences for the state of Piauí, 17 for Caatinga and two for Cerrado. The division Bryophyta presented 25 species, while the Marchantiophyta resulted in 14 species. Fissidentaceae was the most representative among the mosses families, with eight species. For liverworts, the family most abundant was Lejeuneaceae, with 10 species. The substrate rupicolous was the most colonized, followed by corticicolous, terricolous and epixilous. The
\end{abstract}


floristic survey made it possible to expand the geographical distribution of the species for the state of Piauí and between the phytogeographic Domain of Brazil, providing new records for Caatinga and Cerrado.

KEYWORDS: floristic, , liverworts, mosses.

\section{INTRODUÇÃO}

As briófitas representam a linhagem de plantas avasculares terrestres mais antiga da terra, a qual está dividida em três filos distintos: Bryophyta (musgos), Marchantiophyta (hepáticas) e Antocerothophyta (antóceros) (GOFFINET; SHAW, 2009). Estão amplamente distribuídas, sendo mundialmente conhecidas 15.000 espécies (GRADSTEIN et al., 2001). Para o Brasil, estão representadas em 1.524 espécies, distribuídas em 11 antóceros, 633 hepáticas e 880 musgos (COSTA; PERALTA, 2015).

O Nordeste brasileiro é considerado a terceira maior região do país em termos de número espécies de briófitas (COSTA; LUIZI-PONZO, 2010). Atualmente, possui 733 espécies registradas (FLORA DO BRASIL 2020, 2019). O número de publicações sobre a ocorrência de briófitas para região vem crescendo gradualmente nos últimos anos (OLIVEIRA; BASTOS, 2014; SILVA et al., 2014; COSTA et al., 2015; OLIVEIRA; PERALTA, 2015; REIS et al., 2015; SILVA; PÔRTO, 2015; OLIVEIRA; OLIVEIRA, 2016; SILVA et al., 2016; SOUZA et al., 2016; GERMANO et al., 2016; FARIAS et al., 2017; VILAS BÔAS-BASTOS et al., 2017; VIEIRA et al., 2017; VALENTE et al., 2017; COSTA et al., 2018; OLIVEIRA et al., 2018a; OLIVEIRA et al., 2018b; OLIVEIRA et al., 2018c; BATISTA et al., 2018).

Apesar dos avanços em levantamentos brioflorísticos para áreas semiáridas da região Nordeste, o estado do Piauí ainda apresenta sua brioflora bastante desconhecida, com apenas 49 espécies registradas (FLORA DO BRASIL 2020, 2019). Dentre as poucas publicações existentes na literatura, merecem destaque a contribuição dos catálogos de Yano (1989, 1995, 2005, 2006) e o trabalho de Castro et al. (2002), o qual acrescentou 22 espécies de musgos para o Parque Nacional de Sete Cidades.

O estado do Piauí se caracteriza por apresentar extensas áreas de tensão ecológica, com vegetação de transição ou de ecótonos, onde é possível observar um complexo mosaico dos tipos vegetacionais entre Caatinga e manchas de Cerrado caducifólio (CASTRO, 2003; SOUZA et al., 2017). Devido à heterogeneidade de formas vegetais, essas áreas estão sujeitas a apresentar uma grande diversidade de espécies e um número considerável de endemismos, merecendo atenção especial por parte dos pesquisadores (BOTREL et al., 2015; SANTOS et al., 2016; BOLSON, 2018).

O presente trabalho teve como objetivo conhecer a diversidade de musgos e hepáticas ocorrente em uma área de transição entre Caatinga e Cerrado no estado do Piauí, visando ampliar o conhecimento sobre essas plantas no estado.

\section{MATERIAL E MÉTODOS}

O levantamento florístico foi realizado a partir de caminhadas aleatórias em trilhas pré-existentes próximas da Cachoeira do Bota Fora, localizada no município

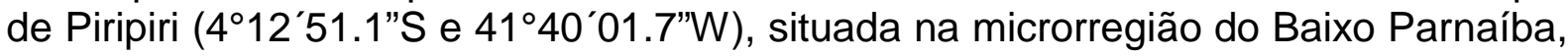
no estado do Piauí (Figura 1). As condições climáticas da região, apresenta clima quente e úmido, com temperaturas variando entre $26^{\circ} \mathrm{C}$ a $38^{\circ} \mathrm{C}$, enquanto que a vegetação compreende uma transição entre cerrado caducifólio e caatinga de várzea (CEPRO, 2005; GENEM, 2017). A precipitação pluviométrica média anual é 
de 1496, $2 \mathrm{~mm}$, com maior concentração nos meses de (janeiro a maio) e menor entre os meses de (julho a dezembro) (RIBEIRO et al., 2015).
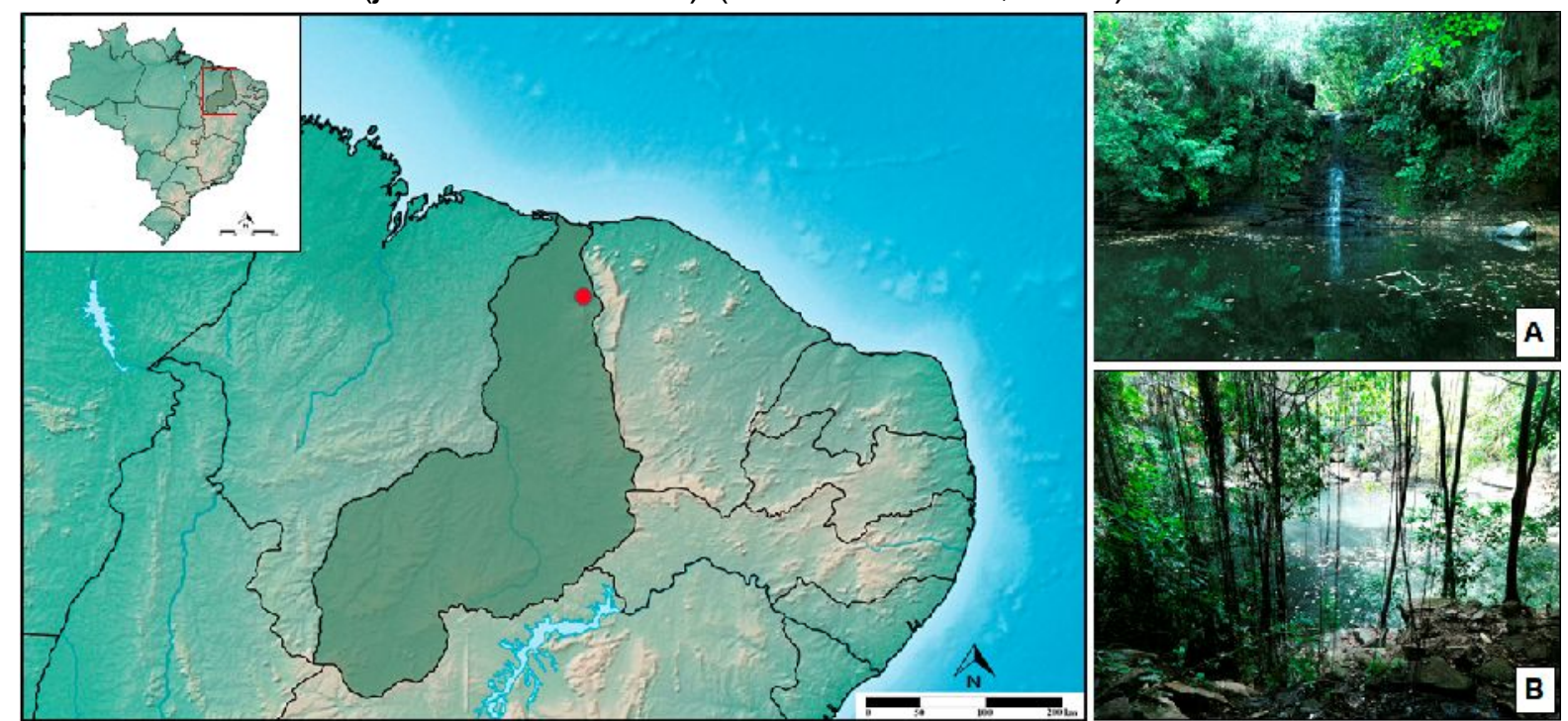

FIGURA 1. Mapa da localização da área de estudo. A. Cachoeira do Bota Fora. B. Vegetação próxima da cachoeira. Fonte: Mapa construído com o software SimpleMappr.

As expedições ocorreram entre o período de outubro de 2017 a fevereiro de 2018, totalizando três visitas ao local. As técnicas de coleta, herborização e preservação dos exemplares foi de acordo com (GRADSTEIN et al., 2001). O material briológico foi coletado em todos os tipos de substratos disponíveis, sendo eles classificados como: corticícolas (tronco vivo), epíxilas (tronco em decomposição), epífilas (superfície de folhas), rupícolas (superfície de rochas) e terrícolas (solo) (ROBBINS, 1952).

A identificação do material, foi realizada a partir das seguintes bibliografias: Gradstein et al. (2001), Gradstein e Costa (2003), Bordin e Yano (2013), Buck (1998), Sharp et al. (1994), Calzadilla e Churchill (2014). O sistema de classificação adotado foi o de Crandall-Stotler et al. (2009) para Marchantiophyta e Goffinet et al. (2009) para Bryophyta.

A distribuição geográfica das espécies foi verificada através do trabalho de Costa et al. (2011), além consultas à plataforma online Flora do Brasil 2020 (2019). A distribuição das espécies foi classificada como ampla (ocorrendo em mais de 10 estados), moderada (5-9) e rara (até 4), conforme a metodologia de (VALENTE e PÔRTO, 2006). Todo o material foi incorporado ao Herbário de Criptógamas da Universidade Estadual do Piauí - HUESPI, Campus Heróis do Jenipapo.

\section{RESULTADOS E DISCUSSÃO}

Foram encontradas 39 espécies de briófitas e uma variedade, distribuídas em 13 famílias e 22 gêneros, das quais 22 são novas ocorrências para o estado do Piauí. Com relação aos Domínios fitogeográficos, 17 são novos registros para a Caatinga e duas são ocorrências novas para o Cerrado. A divisão Bryophyta apresentou 25 espécies, enquanto a divisão Marchantiophyta resultou em 14 espécies, ocorrendo uma predominância de musgos $(65 \%)$ com relação as hepáticas (35\%) (Quadro 1). 
QUADRO 1. Lista das espécies ocorrentes na Cachoeira do Bota Fora, localizada no estado do Piauí, Brasil. Substratos encontrados: Rupícola (RU), Corticícola (CO), Terrícola (TE), Epíxila (EP). Distribuição geográfica com os estados brasileiros. Domínios fitogeográficos: Amazônia (AM), Caatinga (CA), Cerrado (CE), Mata Atlântica (MA), Pampa (PM), Pantanal (PN). Nova ocorrência para Caatinga $\left(\mathrm{CA}^{*}\right)$ e Cerrado $\left(\mathrm{CE}^{*}\right)$. Nova ocorrência para o estado do Piauí $\left({ }^{*}\right)$.

\begin{tabular}{|c|c|c|c|}
\hline $\begin{array}{c}\text { Táxons } \\
\text { (Divisão/Família/Espécie) }\end{array}$ & Substrato & $\begin{array}{c}\text { Distribuição } \\
\text { geográfica no Brasil }\end{array}$ & $\begin{array}{c}\text { Domínio } \\
\text { fitogeográfico }\end{array}$ \\
\hline \multicolumn{4}{|l|}{ BRYOPHYTA } \\
\hline $\begin{array}{c}{ }^{*} \text { Philonotis cernua (Wilson) Griffin } \\
\text { \& W.R.Buck }\end{array}$ & $\mathrm{RU}$ & $\begin{array}{c}\text { CE, MA, PA, SE, PI, DF, } \\
\text { GO, MS, MG, RJ, SP, } \\
\text { PR, RS, SC. }\end{array}$ & CA, CE, MA \\
\hline \multicolumn{4}{|l|}{ Calymperaceae } \\
\hline Calymperes palisotii Schwägr. & CO/EP & $\begin{array}{l}\text { AM, AP, PA, RO, RR, } \\
\text { TO, AL, BA, MA, PB, } \\
\text { PE, PI, RN, SE, GO, } \\
\text { MS, MT, ES, MG, RJ, } \\
\text { SP, PR. }\end{array}$ & $\mathrm{AM}, \mathrm{CA}, \mathrm{CE}, \mathrm{MA}$ \\
\hline Octoblepharum albidum Hedw. & $\mathrm{RU}$ & $\begin{array}{l}\text { AC, AM, AP, PA, RO, } \\
\text { RR, TO, AL, BA, CE, } \\
\text { MA, PB, PE, PI, RR, SE, } \\
\text { DF, GO, MS, MT, ES, } \\
\text { MG, RJ, SP, PR, RS, } \\
\text { SC. }\end{array}$ & $\begin{array}{c}\text { AM, CA, CE, MA, } \\
\text { PM, PN }\end{array}$ \\
\hline Syrrhopodon prolifer Schwägr. & $\mathrm{RU}$ & $\begin{array}{l}\text { AC, AL, AM, BA, CE, } \\
\text { DF, ES, GO, MG, MT, } \\
\text { PA, PE, PR, PI, RJ, RO, } \\
\text { RS, SE, SC, SP, TO. }\end{array}$ & $\mathrm{AM}, \mathrm{CA}, \mathrm{CE}, \mathrm{MA}$ \\
\hline \multicolumn{4}{|l|}{ Fissidentaceae } \\
\hline Fissidens angustifolius Sull. & $\mathrm{RU}$ & $\begin{array}{c}\text { AC, AM, PA, RO, BA, } \\
\text { CE, MA, PA, PB, PI, } \\
\text { GO, RJ, SP, RS. }\end{array}$ & $\begin{array}{c}\text { AM, CE, CA, MA, } \\
\text { PM, PN }\end{array}$ \\
\hline *Fissidens crispus Mont. & $\mathrm{RU}$ & $\begin{array}{c}\text { AM, BA, CE, ES, PE, PI, } \\
\text { DF, GO, MG, MT, PR, } \\
\text { RJ, RO, RR, RS, SC, } \\
\text { SP, TO. }\end{array}$ & $\begin{array}{c}\text { AM, CA, CE, MA, } \\
\text { PM, PN }\end{array}$ \\
\hline${ }^{\star}$ Fissidens flaccidus Mitt. & $\mathrm{RU}$ & $\begin{array}{c}\text { AC, AM, PA, RO, TO, } \\
\text { BA, CE, MA, PB, PE, PI, } \\
\text { SE, DF, GO, MS, MT, } \\
\text { ES, MG, RJ, SP, PR, } \\
\text { RS. }\end{array}$ & $\begin{array}{c}\text { AM, CA, CE, MA, } \\
\text { PM, PN }\end{array}$ \\
\hline Fissidens goyazensis Broth. & $\mathrm{RU}$ & $\begin{array}{l}\text { AM, BA, CE, PB, PE, PI, } \\
\text { DF, GO, MG, RJ, SP. }\end{array}$ & $\mathrm{AM}, \mathrm{CA}, \mathrm{CE}, \mathrm{MA}$ \\
\hline${ }^{\star}$ Fissidens neglectus H.A. Crum & $\mathrm{RU}$ & MA, MG, PE, PI, SP. & $\mathrm{CE}, \mathrm{MA}, \mathrm{CA}^{*}$ \\
\hline Fissidens radicans Mont. & $\mathrm{RU}$ & $\begin{array}{l}\mathrm{PA}, \mathrm{BA}, \mathrm{MA}, \mathrm{PB}, \mathrm{SE}, \mathrm{PI}, \\
\text { DF, ES, MG, SP. }\end{array}$ & $\mathrm{AM}, \mathrm{CE}, \mathrm{MA}, \mathrm{CA}$ \\
\hline Fissidens submarginatus Bruch & TE & $\begin{array}{l}\mathrm{AC}, \mathrm{AM}, \mathrm{PA}, \mathrm{RO}, \mathrm{BA} \\
\mathrm{CE}, \mathrm{MA}, \mathrm{PB}, \mathrm{PE}, \mathrm{PI}\end{array}$ & $\begin{array}{c}\mathrm{AM}, \mathrm{CA}, \mathrm{CE}, \mathrm{MA}, \\
\text { PM, PN }\end{array}$ \\
\hline
\end{tabular}




\begin{tabular}{|c|c|c|c|}
\hline $\begin{array}{c}\text { Táxons } \\
\text { (Divisão/Família/Espécie) }\end{array}$ & Substrato & $\begin{array}{c}\text { Distribuição } \\
\text { geográfica no Brasil }\end{array}$ & $\begin{array}{c}\text { Domínio } \\
\text { fitogeográfico }\end{array}$ \\
\hline & & $\begin{array}{l}\text { RN, DF, GO, MT, ES, } \\
\text { MG, RJ, SP, RS, SC. }\end{array}$ & \\
\hline${ }^{*}$ Fissidens zollingeri Mont. & $\mathrm{RU} / \mathrm{TE}$ & $\begin{array}{c}\text { AC, AM, PA, RO, RR, } \\
\text { TO, AL, BA, CE, PB, } \\
\text { PE, PI, MA, SE, DF, } \\
\text { GO, MS, MT, ES, MG, } \\
\text { RJ, SP, PR, RS, SC. }\end{array}$ & $\begin{array}{c}\text { AM, CA, CE, MA, } \\
\text { PN }\end{array}$ \\
\hline $\begin{array}{c}\text { Hypnaceae } \\
\text { *Isopterygium tenerifolium Mitt. }\end{array}$ & TE/CO & $\begin{array}{l}\text { AM, PA, RO, BA, CE, } \\
\text { PI, DF, GO, MG, MT, } \\
\text { PR, RJ, PR, RS, SC, } \\
\text { SP. }\end{array}$ & $\mathrm{AM}, \mathrm{CE}, \mathrm{MA}, \mathrm{CA}^{*}$ \\
\hline $\begin{array}{c}\text { Vesicularia vesicularis (Schwägr.) } \\
\text { Broth. }\end{array}$ & $\mathrm{RU}$ & $\begin{array}{l}\text { AC, AM, AP, PA, RO, } \\
\text { RR, TO, BA, MA, PE, } \\
\text { PI, GO, MS, MT, ES, } \\
\text { MG, RJ, SP, PR, RS, } \\
\text { SC. }\end{array}$ & 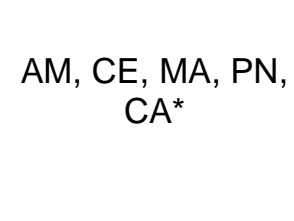 \\
\hline $\begin{array}{l}\text { *Vesicularia vesicularis var. } \\
\text { portoricensis (Brid.) W.R.Buck }\end{array}$ & $\mathrm{RU}$ & $\begin{array}{l}\text { AM, AP, BA, PE, PI, } \\
\text { GO, MS, MT, MG, RJ, } \\
\text { SP, PR, SC. }\end{array}$ & 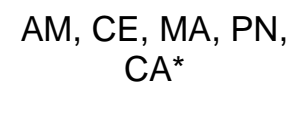 \\
\hline $\begin{array}{c}\text { Pilotrichaceae } \\
{ }^{*} \text { Callicostella merkelii (Hornsch.) } \\
\text { A.Jaeger }\end{array}$ & $\mathrm{RU}$ & $\begin{array}{l}\text { AC, AM, BA, CE, PI, } \\
\text { GO, MG, PA, PE, RO, } \\
\text { SC, SP. }\end{array}$ & $\mathrm{AM}, \mathrm{CE}, \mathrm{MA}, \mathrm{CA}^{*}$ \\
\hline $\begin{array}{c}{ }^{*} \text { Callicostella pallida (Hornsch.) } \\
\text { Ångström }\end{array}$ & $\mathrm{RU}$ & $\begin{array}{l}\text { AC, AL, AM, AP, BA, } \\
\text { CE, PI, DF, ES, GO, } \\
\text { MG, MS, MT, PA, PE, } \\
\text { PR, RJ, RO, RS, SC, } \\
\text { SP, TO. }\end{array}$ & $\begin{array}{c}\text { AM, CA, CE, MA, } \\
\text { PM, PN }\end{array}$ \\
\hline Pottiaceae & & & \\
\hline $\begin{array}{l}\text { Hyophilla involuta (Hook.) } \\
\text { A.Jaeger }\end{array}$ & RU & $\begin{array}{l}\text { AM, PA, RO, RR, AL, } \\
\text { BA, CE, MA, PB, PE, PI, } \\
\text { DF, GO, MS, MT, ES, } \\
\text { MG, RJ, SP, PR, RS. }\end{array}$ & $\begin{array}{c}\text { AM, CA, CE, MA, } \\
\text { PM, PN }\end{array}$ \\
\hline $\begin{array}{c}\text { Sematophyllaceae } \\
\text { Microcalpe subsimplex (Hedw.) } \\
\text { Spruce }\end{array}$ & $\mathrm{RU} / \mathrm{CO} / \mathrm{EP}$ & $\begin{array}{l}\text { AC, AM, AP, PA, RO, } \\
\text { RR, TO, AL, BA, CE, } \\
\text { MA, PE, PI, SE, MT, DF, } \\
\text { GO, MS, ES, MG, RJ, } \\
\text { SP, PR, RS, SC. }\end{array}$ & AM, AT, CA, CE \\
\hline $\begin{array}{l}\text { Trichosteleum subdemissum } \\
\text { (Besch.) A.Jaeger }\end{array}$ & $\mathrm{CO}$ & $\begin{array}{l}\text { AM, PA, RO, RR, BA, } \\
\text { MA, PI, DF, GO, MT, } \\
\text { MG, RJ, SP. }\end{array}$ & $\mathrm{AM}, \mathrm{CE}, \mathrm{MA}, \mathrm{CA}^{*}$ \\
\hline $\begin{array}{c}\text { Vitalia galipensis (Müll. Hal.) } \\
\text { P.E.A.S.Câmara, Carv.-Silva \& } \\
\text { W.R.Buck } \\
\text { Stereophyllaceae }\end{array}$ & $\mathrm{RU} / \mathrm{EP}$ & $\begin{array}{l}\text { BA, ES, GO, MG, MT, } \\
\text { PA, PE, PR, RJ, RO, } \\
\text { RR, RS, SC, SP, TO. }\end{array}$ & AM, CE, MA \\
\hline $\begin{array}{l}\text { Entodontopsis leucostega (Brid.) } \\
\text { W.R.Buck \& Ireland }\end{array}$ & $\mathrm{RU} / \mathrm{TE}$ & $\begin{array}{l}\text { AC, AM, PA, RR, RO, } \\
\text { TO, BA, CE, MA, PB, } \\
\text { PE, PI, DF, GO, MS, } \\
\text { MT, MG, RJ, SP. }\end{array}$ & $\begin{array}{c}\text { AM, CA, CE, MA, } \\
\text { PN }\end{array}$ \\
\hline
\end{tabular}




\begin{tabular}{|c|c|c|c|}
\hline $\begin{array}{c}\text { Táxons } \\
\text { (Divisão/Família/Espécie) }\end{array}$ & Substrato & $\begin{array}{c}\text { Distribuição } \\
\text { geográfica no Brasil }\end{array}$ & $\begin{array}{c}\text { Domínio } \\
\text { fitogeográfico }\end{array}$ \\
\hline $\begin{array}{l}\text { Entodontopsis nitens (Mitt.) } \\
\text { W.R.Buck \& Ireland }\end{array}$ & $\mathrm{RU}$ & $\begin{array}{l}\text { AC, PA, AL, BA, CE, } \\
\text { PE, GO, MS, MT, MG, } \\
\text { SP, PR, RS. }\end{array}$ & $\begin{array}{c}\mathrm{AM}, \mathrm{CE}, \mathrm{MA}, \mathrm{PN} \\
\mathrm{CA}^{*}\end{array}$ \\
\hline $\begin{array}{l}{ }^{*} \text { Entodontopsis panamensis } \\
\text { (E.B.Bartram) W.R.Buck \& Ireland }\end{array}$ & $\mathrm{RU}$ & $\mathrm{BA}, \mathrm{PI}, \mathrm{RO}$. & $\mathrm{AM}, \mathrm{MA}, \mathrm{CA}^{*}, \mathrm{CE}^{*}$ \\
\hline $\begin{array}{l}\text { *Eulacophyllum cultelliforme } \\
\text { (Sull.) W.R.Buck \& Ireland }\end{array}$ & $\mathrm{RU}$ & $\begin{array}{l}\text { AM, TO, BA, MA, PB, } \\
\text { PE, PI, MS, MT, ES, } \\
\text { MG, RJ, SP. }\end{array}$ & $\mathrm{AM}, \mathrm{MA}, \mathrm{CE}, \mathrm{CA}^{*}$ \\
\hline \multicolumn{4}{|l|}{$\begin{array}{c}\text { MARCHANTIOPHYTA } \\
\text { Aneuraceae }\end{array}$} \\
\hline $\begin{array}{l}{ }^{*} \text { Riccardia cataractarum (Spruce) } \\
\text { Schiffn. }\end{array}$ & TE/RU & $\begin{array}{l}\text { BA, CE, PI, ES, GO, } \\
\text { MG, MS, MT, RJ, RS, } \\
\text { SC, SP. }\end{array}$ & $\mathrm{CE}, \mathrm{MA}, \mathrm{PN}, \mathrm{CA}^{*}$ \\
\hline Corsiniaceae & & & \\
\hline $\begin{array}{l}\text { Cronisia weddellii (Mont.) Grolle } \\
\text { Fossombroniaceae }\end{array}$ & $\mathrm{RU}$ & $\begin{array}{l}\text { TO, AL, BA, CE, MA, } \\
\text { PE, PI, GO, MT, ES. }\end{array}$ & CA, CE, MA \\
\hline $\begin{array}{c}\text { Fossombronia porphyrorhiza } \\
\text { (Nees) Prosk. }\end{array}$ & $\mathrm{RU}$ & $\begin{array}{l}\text { BA, CE, DF, ES, GO, } \\
\text { MA, MG, MS, MT, PE, } \\
\text { PI, RJ, SP, TO. }\end{array}$ & CA, CE, MA, PN \\
\hline $\begin{array}{c}\text { Lejeuneaceae } \\
\text { *Acrolejeunea torulosa (Lehm. \& } \\
\text { Lindenb.) Schiffn. }\end{array}$ & $\mathrm{CO} / \mathrm{EP}$ & $\begin{array}{l}\text { AC, AL, AM, BA, PI, ES, } \\
\text { GO, MA, MG, MS, MT, } \\
\text { PA, PE, RJ, RO, RR, } \\
\text { SP, PR, RS. }\end{array}$ & $\mathrm{AM}, \mathrm{CE}, \mathrm{MA}, \mathrm{CA}^{*}$ \\
\hline $\begin{array}{l}{ }^{*} \text { Cheilolejeunea discoidea (Lehm. } \\
\text { \& Lindenb.) Kachr. \& R.M.Schust. }\end{array}$ & CO/EP & $\begin{array}{l}\text { BA, PI, DF, ES, GO, } \\
\text { MG, MS, MT, PA, SE, } \\
\text { SP. }\end{array}$ & $\mathrm{CE}, \mathrm{MA}, \mathrm{PN}, \mathrm{CA}^{*}$ \\
\hline $\begin{array}{c}{ }^{*} \text { Cololejeunea minutissima (Sm.) } \\
\text { Schiffn. }\end{array}$ & $\mathrm{RU}$ & $\begin{array}{l}\text { AC, AM, BA, PI, ES, } \\
\text { MG, RJ, MS, MT, PE, } \\
\text { PR, SC, SP. }\end{array}$ & $\begin{array}{c}\mathrm{AM}, \mathrm{CE}, \mathrm{MA}, \mathrm{PN} \\
\mathrm{CA}^{*}\end{array}$ \\
\hline $\begin{array}{l}{ }^{*} \text { Dibrachiella parviflora (Nees) } \\
\text { X.Q. Shi, R.L. Zhu \& Gradst. }\end{array}$ & $\mathrm{RU}$ & $\begin{array}{c}\text { AM, BA, PE, PI, RO, } \\
\text { SP. }\end{array}$ & $\mathrm{AM}, \mathrm{MA}, \mathrm{CA}^{*}$ \\
\hline *Lejeunea aphanes Spruce & $\mathrm{CO}$ & BA, CE, PI. & $\mathrm{MA}, \mathrm{CA}^{*}$ \\
\hline $\begin{array}{c}\text { *Lejeunea caulicalyx (Steph.) } \\
\text { E.Reiner \& Goda }\end{array}$ & $\mathrm{CO}$ & $\begin{array}{l}\text { AC, PA, RR, AL, BA, } \\
\text { PE, PI, MS, MT, ES, RJ, } \\
\text { SP, PR. }\end{array}$ & $\begin{array}{c}\mathrm{AM}, \mathrm{CE}, \mathrm{MA}, \mathrm{PN} \\
\mathrm{CA}^{*}\end{array}$ \\
\hline${ }^{*}$ Lejeunea flava (Sw.) Nees & $\mathrm{RU}$ & $\begin{array}{c}\text { AC, AL, AM, BA, CE, PI, } \\
\text { DF, ES, GO, MA, MG, } \\
\text { MS, MT, PA, PE, PR, } \\
\text { RJ, RO, RR, RS, SC, } \\
\text { SP, TO. }\end{array}$ & $\begin{array}{l}\text { AM, CA, CE, MA, } \\
\text { PM, PN }\end{array}$ \\
\hline *Lejeunea immersa Spruce & $\mathrm{CO} / \mathrm{EP} / \mathrm{RU}$ & $\begin{array}{l}\text { AM, RO, AL, BA, CE, } \\
\text { PE, PI, RJ, SP. }\end{array}$ & $\mathrm{MA}, \mathrm{CA}^{*}$ \\
\hline $\begin{array}{c}\text { *Lejeunea grosiretis (Steph.) } \\
\text { E.Reiner \& Goda }\end{array}$ & $\mathrm{CO} / \mathrm{RU}$ & $\mathrm{BA}, \mathrm{PI}, \mathrm{RJ}, \mathrm{SP}$ & $\mathrm{MA}, \mathrm{CA}^{*}$ \\
\hline $\begin{array}{c}\text { *Lejeunea laetevirens Nees \& } \\
\text { Mont. }\end{array}$ & $R U$ & $A C, A L, A M, A P, B A$, & $\begin{array}{c}\text { AM, CA, CE, MA, } \\
\text { PN }\end{array}$ \\
\hline
\end{tabular}




\begin{tabular}{cccc}
\hline $\begin{array}{c}\text { Táxons } \\
\text { (Divisão/Família/Espécie) }\end{array}$ & Substrato & $\begin{array}{c}\text { Distribuição } \\
\text { geográfica no Brasil }\end{array}$ & $\begin{array}{c}\text { Domínio } \\
\text { fitogeográfico }\end{array}$ \\
\hline & & CE, PI, DF, ES, GO, \\
& & MA, MS, MT, PA, PB, \\
Ricciaceae & PE, PR, RJ, RN, RR, & \\
& & RS, SC, SE, SP. & \\
${ }^{*}$ Riccia brasiliensis Schiffn. & TE & PE, PI, SP, PR, RS, SC. & MA, CA* \\
\hline
\end{tabular}

A Família Fissidentaceae foi a mais representativa entre os musgos, tendo o seu único Gênero Fissidens, como o mais diversificado para divisão, com oito espécies. Esse Gênero representa um dos mais representativos em termos de número de espécies para região neotropical (GRADSTEIN et al., 2001). Em relação às hepáticas, a família mais abundante foi Lejeuneaceae, com 10 espécies, das quais seis pertencem ao Gênero Lejeunea. Segundo Bastos e Yano (2009), este é considerado o maior e mais complexo gênero da família.

Quanto aos substratos colonizados, o predominante foi o rupícolo com (75\%) das espécies crescendo sobre ele, seguido de corticícolo (27,5\%), terrícolo (15\%) e epíxilo (15\%). A alta frequência de briófitas rupícolas está relacionada com a grande disponibilidade de superfícies rochosas na área de estudo, próximas as margens da cachoeira ou em locais sombreados. A ausência de espécies epífilas pode ser justificada pelo fato de que a vegetação existente na região apresenta características típicas de ambientes semiáridos, onde em resposta ao estresse hídrico, os indivíduos desenvolvem adaptações como folhas pequenas ou perda das folhas durante os longos períodos de estiagem (MEIRA et al., 2017; FERNANDES; QUEIROZ, 2018).

A alta frequência de musgos pode está relacionada com sua complexidade estrutural e fisiológica, o que os torna resistentes às condições ambientais de áreas semiáridas, como longos períodos de seca e baixa umidade (PÔRTO et. al., 1994). Esse resultado também condiz com o encontrado em outras áreas de Caatinga e Cerrado do Nordeste (SILVA et al., 2014; COSTA et. al., 2015; BATISTA; SANTOS, 2018; OLIVEIRA et al., 2018b).

Ao comparar os resultados obtidos com outros levantamentos brioflorísticos realizados para Caatinga e Cerrado, nota-se que (36\%) das espécies encontradas estão em comum com o estudo de Castro et al. (2002), realizado no Parque Nacional de Sete Cidades, localizada no estado do Piauí. Também há correspondência de $30 \%$ das espécies encontradas com a pesquisa de Pôrto e Bezzera (1996) e de 12\% com a de Pôrto et al. (1994), ambas realizadas no estado de Pernambuco, em ambientes semiáridos.

A maioria das espécies registradas está amplamente distribuída entre os estados brasileiros (82\%), ocorrendo em mais de 10 estados. A ampla distribuição territorial pode ser explicada devido ao grupo dispor de diferentes estratégias de vida, incluindo a forma de dispersão que lhes permite atingir longas distâncias (GLIME, 2017). Cerca de 10\% das espécies apresenta distribuição moderada e $8 \%$ possui distribuição restrita em até quatro estados.

O inventário florístico também possibilitou ampliar a distribuição geográfica das espécies entre os domínios fitogeográficos do Brasil, fornecendo novos registros para Caatinga e Cerrado, com destaque em: Entodontopsis panamensis (E.B.Bartram) W.R.Buck \& Ireland, que antes só possuia registro para a Mata Atlântica e Floresta amazônica; e Lejeunea aphanes Spruce, L. immersa Spruce, L. 
grosiretis (Steph.) E.Reiner \& Goda e Riccia brasiliensis Schiffn., registradas apenas na Mata Atlântica.

\section{CONCLUSÃO}

O elevado número de novos registros encontrados ressalta a importância de realizar novos inventários florísticos em áreas semiáridas como as da Caatinga e Cerrado, com intuito de ampliar de ampliar o conhecimento as espécies de briófitas ocorrentes no estado do Piauí e região Nordeste do Brasil.

\section{AGRADECIMENTOS}

Ao Programa Institucional de Bolsas de Iniciação Cientifica (PIBIC) da Universidade Estadual do Piauí (UESPI) pelo apoio financeiro referente à bolsa concedida à primeira autora.

\section{REFERÊNCIAS}

BASTOS, C.J.P. \& YANO, O. O gênero Lejeunea Libert (Lejeuneaceae) no Estado da Bahia, Brasil. Hoehnea, v.36, n.2, p.303-320, 2009. Disponível em: <http://dx.doi.org/10.1590/S2236-89062009000200008>. DOI: 10.1590/S223689062009000200008.

BATISTA, W.V.S.M.; PÔRTO, K.C. \& SANTOS, N.D. Distribution, ecology, and reproduction of bryophytes in a humid enclave in the semiarid region of northeastern Brazil. Acta Botanica Brasilica, v.32, n.2, p.303-313, 2018. Disponível em: <http://dx.doi.org/10.1590/0102-33062017abb0339>. DOI: 10.1590/0102$33062017 \mathrm{abb0339.}$

BOTREL, R. T.; BRITO, D.R.S; SOUSA, W.C.; SOUZA, A.M.; HOLANDA, A.C. Fenologia de uma espécie arbórea em ecótono Caatinga / Cerrado no sul do Piauí. Revista Verde de Agroecologia e desenvolvimento Sustentável, v.10, n.3, p.7, 2015. Disponível em: <http://dx.doi.org/10.18378/rvads.v10i3.3587>. DOI: 10.18378/rvads.v10i3.3587.

BORDIN, J. \& YANO, O. Fissidentaceae (Bryophyta) do Brasil. São Paulo: Boletim do Instituto de Botânica, 2013,168p.

BOLSON, S.H. O Cerrado nas metas brasileiras do acordo de Paris: A Omissão do Estado Brasileiro com o desmatamento da cumeeira da américa do sul . Revista de Direito Ambiental E Socioambientalismo, v.4, n.1, p.112-131, 2018. Disponível em: <http://dx.doi.org/10.26668/IndexLawJournals/2525-9628/2018.v4i1.3996>. DOI: 10.26668/IndexLawJournals/2525-9628/2018.v4i1.3996

BUCK, W. R. Pleurocarpous Mosses of the West Indies. New York: Memoirs of The New York Botanical Garden,1998, 400p.

CASTRO, A.A.J.F. Biodiversidade e riscos antrópicos no Nordeste do Brasil. Territorium, v. 10, p.4560, 2003. Disponível em: <https://doi.org/10.14195/16477723>. DOI: 10.14195/1647-7723.

CASTRO, N.M.C.F.; PÔRTO, K.C.; YANO, O. \& CASTRO, A.A.J.F. Levantamento Florístico de Bryopsida de Cerrado e Mata Ripícola do Parque Nacional de Sete Cidades, Piauí, Brasil. Acta Botanica Brasilica, v.16, n.1, p.61-76, 2002. Disponível 
em: <http://dx.doi.org/10.1590/S0102-33062002000100008>. DOI: 10.1590/S010233062002000100008.

CALZADILLA, E.; CHURCHILL, S. Glosario ilustrado para musgos neotropicales. Bolivia: Missouri Botanical Garden, Museo de História Natural, 2014, 122p.

COSTA, D.P., PÔRTO, K.C., LUIZI-PONZO, A.P., ILKIU-BORGES, A.L., BASTOS, C.J.P., et al.; Synopsis of the Brazilian moss flora: checklist, distribution and conservation. Nova Hedwigia, v.93, n.3-4, p.277-334, 2011. Disponível em: <https://dx.doi.org/10.1127/0029-5035/2011/0093-0277>. DOI: 10.1127/00295035/2011/0093-0277.

COSTA, D.P.; PERALTA, D.F. Bryophytes diversity in Brazil. Rodriguésia, v.66, n.4, p.1063-1071, 2015. Disponivel em: <http://dx.doi.org/10.1590/21757860201566409>. DOI: 10.1590/2175-7860201566409.

COSTA, A.M.R.; OLIVEIRA, R.R.; SANTOS-SILVA, D.L.; SÁ, N.A.S.; CONCEIÇÃO, G.M. Briófitas do Cerrado Maranhense, Nordeste do Brasil. NBC-Periódico Científico do Núcleo de Biociências, v. 8, n. 16, p.33-45, 2018. Disponível em: <https://www.metodista.br/revistas/revistas-izabela/index.php/bio/article/view/1698>. Acesso em: 15 de Dez. 2019.

COSTA, F.B.; SILVA, E.O.; CONCEIÇÃO, G.M. Hepáticas (Marchantiophyta) e musgos (Bryophyta) da Área de Proteção Ambiental do Buriti do Meio, município de Caxias, Maranhão, Brasil. Scientia Plena, v.11, p.1-4, 2015. Disponível em: $<$ https://www.scientiaplena.org.br/sp/article/view/1959/0>. Acesso em: 20 de Jan. 2019.

CRANDALL-STOTLER, B.; STOTLER, R. E.; LONG, D. G. Morphology and classification of the Marchantiophyta. In: GOFFINET, B.; SHAW, A. J. Bryophyte Biology. 2. ed. New Yourk: Cambdrige University Press, 2009,1-54p.

FARIAS, R.S.; SILVA, M.P.P.; MACIEL-SILVA, A.S.; PÔRTO, K.C. Influence of environmental factors on the distribution of Calymperes and Syrrhopodon (Calymperaceae, Bryophyta) in the Atlantic Forest of Northeastern Brazil. Flora, v. 234, p. 158-164, 2017. Disponível em: <https://doi.org/10.1016/j.flora.2017.07.011>. DOI: 10.1016/j.flora.2017.07.011.

FERNANDES, M.F.; QUEIROZ, L.P. Vegetação e flora da Caatinga. Ciência e Cultura, v.70, n.4, p.51-56, 2018. Disponível em: <http://dx.doi.org/10.21800/231766602018000400014>. DOI: 10.21800/2317-66602018000400014.

Fundação Cepro e Ministério das Minas e Energia (MME). Diagnóstico do Setor Mineral do Estado do Piauí. Teresina: Fundação Centro de Pesquisa Econômicas e Sócias, 2005. Disponível em: <http://www.cepro.pi.gov.br/download/200804/CEPRO16_6695f7c23c.pdf>. Acesso em: 19 de Jan. 2019.

FLORA DO BRASIL 2020. Jardim Botânico do Rio de Janeiro. Disponível em:<http://floradobrasil.jbrj.gov.br/ >. Acesso em: 15 de Jan. 2019.

GENEM, R.S. Caatinga: Estratégias de conservação. Brasília: Consultoria Legislativa da Câmara dos Deputados, 2017. Disponível em: $<$ http://bd.camara.gov.br/bd/bitstream/handle/bdcamara/34479/caatinga_roseli_gane m.pdf?sequence=5>. Acesso em: 17 de Fev. 2019. 
GERMANO. S.R.; SILVA, J.B.; PERALTA, D.F. Paraíba State, Brazil: a hotspot of bryophytes. Phytotaxa, v.259, n.3, p.251-278, 2016. Disponível em: <http://dx.doi.org/10.11646/phytotaxa.258.3.2>. DOI: 10.11646/phytotaxa.258.3.2.

GLIME, J. M. Capítulo 1 - Introdução. In: Bryophyte Ecology Ebook. Michigan Technological University and the International Association of Bryologists. v.1, 2017. Disponível em: <http://www.bryoecol.mtu.edu/> Acesso em: 28 de Dez. 2018.

GOFFINET, B.; BUCK, W. R.; SHAW, A. J. Morphology and classification of the Bryophyta. In: GOFFINET, B.; SHAW, A. J. Bryophyte Biology. 2. ed. New Yourk: Cambridge University Press, 2009. 55-138p.

GOFFINET, B.; SHAW, A.J. Bryophyte Biology. 2. ed. New Yourk: Cambridge University Press, 2009, 580p.

GRADSTEIN, S.R.; COSTA, D.P. The Hepaticae and Anthocerotae of Brazil. New Yourk: Memoirs of The New York Botanical Garden, 2003, v.87, 318p.

GRADSTEIN, S. R.; CHURCHILL, S. P.; SALAZAR-ALLEN, N. Guide to the Bryophytes of Tropical America. New York: Memoirs of The New York Botanical Garden, 2001, 577p.

MEIRA, M.M.C.; SILVA, C.R.T.; CARVALHO, L.O.; BRASIL, M.D.; RODRIGUES, M.A.; SANTOS, P.A. A BELEZA SECA: Aspectos do paisagismo no semiárido brasileiro. Mix Sustentável, v.3, n.2, p.108-113, 2017. Disponível em: <https://doi.org/10.29183/2447-3073.MIX2017.v3.n2.108-113>. DOI: 10.29183/24473073.MIX2017.v3. n2.108-113.

OLIVEIRA, H.C.; BASTOS, C. J. P. Briófitas epífitas de fragmentos de Floresta Atlântica da Reserva Ecológica Michelin, Estado da Bahia, Brasil. Hoehnea, v.41, p.631-645, 2014. Disponível em: <http://dx.doi.org/10.1590/2236-8906-35/2013>. DOI: 10.1590/2236-8906-35/2013.

OLIVEIRA, H.C.; PERALTA, D.F. Adições a brioflora de musgos acrocárpicos (Bryophyta) do Estado do Ceará, Brasil. Pesquisas. Botânica, v.67, p.37-50, 2015. Disponível em: <http://anchietano.unisinos.br/publicacoes/botanica/botanica67/003.pdf>. Acesso em: 11 de Fev. 2019.

OLIVEIRA, H.C.; OLIVEIRA, S. M. Vertical distribution of epiphytic bryophytes in Atlantic Forest fragments in northeastern Brazil. Acta Botanica Brasilica, v.30, n.4, p.609-617, 2016. Disponível em: <http://dx.doi.org/10.1590/010233062016abb0303>. DOI: 10.1590/0102-33062016abb0303.

OLIVEIRA, R.F.; SILVA, G.S.; OLIVEIRA, R.R.; OLIVEIRA, H.C.; CONCEICAO, G.M. Musgos (Bryophyta) de um fragmento de cerrado maranhense, Brasil. Biota Amazônia, v.8, n.2, p.12-18, 2018a. Disponível em: <http://dx.doi.org/10.18561/21795746>. DOI: 10.18561/21795746.

OLIVEIRA, R.R.; MENDEIROS, D.L.; OLIVEIRA, H.C.; CONCEIÇÃO, G.M. Briófitas de área sob o domínio fitogeográfico do Cerrado e novas ocorrências para o Maranhão e região Nordeste do Brasil. Iheringia Série Botânica, v.73, n.2, p.1-195, 2018b. Disponível em: <http://dx.doi.org/10.21826/2446-8231201873211>. DOI: $10.21826 / 2446-8231201873211$. 
OLIVEIRA, R.R.; OLIVEIRA, H.C.; PERALTA, D.F.; CONCEIÇÃO, G.M. Acrocarpic mosses (Bryophyta) of Chapada das Mesas National Park, Maranhão, Brazil. Check List, v.14, n.6, p.967-975, 2018c.Disponível em: <https://doi.org/10.15560/14.6.967>. DOI:10.15560/14.6.967.

PÔRTO, K.C.; SILVEIRA, M.F.G.; SÁ, P.S.A. Briófitas da Caatinga I. Estação experimental do Ipa, Caruaru - PE. Acta Botanica Brasilica, v.8, n.1, 1994. Disponível em: <http://dx.doi.org/10.1590/S0102-33061994000100008>. DOI: 10.1590/S0102-33061994000100008.

PÔRTO, K. C.; BEZERRA, M.F. Briófitas de caatinga: 2. Agrestina, Pernambuco, Brasil. Acta Botanica Brasilica, v.10, n.1, p.93-102,1996. Disponível em <http://dx.doi.org/10.1590/S0102-33061996000100009>. DOI: 10.1590/S010233061996000100009.

REIS, L. C.; OLIVEIRA, H.C.; BASTOS, C.J.P. Hepáticas (Marchantiophyta) epífitas de duas áreas de Floresta Atlântica no Estado da Bahia, Brasil. Pesquisas.

Botânica, v.67, p.225-241, 2015. Disponível em:
<http://www.anchietano.unisinos.br/publicacoes/botanica/botanica67/015.pdf>. Acesso em: 10 de Jan. 2019.

RIBEIRO, A.A.; SIMÃO, M.; SANTOS, A.R.B. Balanço hídrico climatológico para os municípios de Piripiri e São João do Piauí, Piauí, Brasil. Brazilian Journal of Biosystems Engineering, v.9, n.3, p.228-235, 2015. Disponível em: <http://dx.doi.org/10.18011/bioeng2015v9n3p228235>.

DOI: 10.18011/bioeng2015v9n3p228-235.

ROBBINS, R.G. Bryophyte Ecology of a Dune Area in New Zealand. Acta Geobotanica, v.4, p.1-31, 1952. Disponível em: $<$ https://doi.org/10.1007/BF00452923>. DOI: 10.1007/BF00452923.

SANTOS, A.M.A.; NASCIMENTO, D.P.B.; COLAÇO, M.A.S.; SILVA, S.S. Reflexões sobre os efeitos das Mudanças Climáticas na Biodiversidade da Caatinga. Diversitas Journal, v.1, n.1, p.113-118, 2016. Disponível em: < https://doi.galoa.com.br/doi/10.17648/diversitas-journal-v1i1.218>.

DOI: 10.17648/diversitas-journal-v1i1.218.

SHARP, A.J.; CRUM, H.; ECKEL, P. The Moss Flora of Mexico. New Yourk: Memoirs of The New York Botanical Garden, 1994, v.69, p.1-1113.

SILVA, M.P.P.; PÔRTO, K.C. Diversity of bryophytes in priority areas for conservation in the Atlantic forest of northeast Brazil. Acta Botanica Brasilica, v.29, n.1, p.16-23, 2015. Disponível em: <http://dx.doi.org/10.1590/010233062014abb3534>. DOI: 10.1590/0102-33062014abb3534.

SILVA, M.P.P.; ZICKEL, C.S.; PÔRTO, K.C.K. Bryophyte communities of restingas in Northeastern Brazil and their similarity to those of other restingas in the country. Acta Botanica Brasilica, v.30, n.3, p.455-461, 2016. Disponível em: <http://dx.doi.org/10.1590/0102-33062016abb0130>. DOl:10.1590/0102$33062016 \mathrm{abb} 0130$.

SILVA, T.O.; SILVA, M.P.P.; PÔRTO, K.C. Briófitas de Afloramentos Rochosos do Estado de Pernambuco, Brasil. Boletim do Museu de Biologia Mello Leitão, v.36, 
p.85-100, 2014. Disponível em: <http://repositorio.ufba.br/ri/handle/ri/17409>. Acesso em: 11 de Jan. 2019.

SOUZA, M.P.; COUTINHO, J.M.C.P.; SILVA, L.S.; AMORIM, F.S.; ALVES, A.R. Composição e estrutura da vegetação de caatinga no sul do Piauí, Brasil. Revista Verde de Agroecologia e Desenvolvimento Sustentável, v.12, n.2, p.210-217, 2017. Disponível em: DOI: <http://dx.doi.org/10.18378/rvads.v12i2.4588>. DOI: 10.18378/rvads.v12i2.4588.

SOUZA, A.M.; VALENTE, E.B.; BASTOS, C.J.P.; AZEVEDO, C.O. Marchantiophyta da Reserva do Poço Escuro, Vitória da Conquista, Bahia, Brasil. Natureza online, v.14, p.64, 2016. Disponível em: <http://www.naturezaonline.com.br/natureza/conteudo/pdf/NOL20160102.pdf>.

Acesso em: 27 de Jan. 2019.

VALENTE, E.B.; PÔRTO, K.C. Hepáticas (Marchantiophyta) de um fragmento de Mata Atlântica na Serra da Jibóia, município e Santa Teresinha, Bahia, Brasil. Acta Botanica Brasilica, v.20, n.2, p.433-441, 2006. Disponível em: <http://dx.doi.org/10.1590/S0102-33062006000200018>. DOI:10.1590/S010233062006000200018.

VALENTE, B.E.; PÔRTO, C.K.; BASTOS, C.J.P. Habitat heterogeneity and diversity of bryophytes in campos rupestres. Acta Botanica Brasilica, v.31, n.2, p.241-249, 2017. Disponível em: <http://dx.doi.org/10.1590/0102-33062016abb0450>. DOI: 10.1590/0102-33062016abb0450.

VILAS BÔAS-BASTOS, S.B.; BASTOS, C. J. P.; Costa, K.R. Brioflora da Área de Relevante Interesse Ecológico Serra do Orobó, municípios de Ruy Barbosa e Itaberaba, Bahia, Brasil. Pesquisas. Botânica, v. 70, p. 79-98, 2017. Disponível em: <http://www.anchietano.unisinos.br/publicacoes/botanica/botanica.htm>. Acesso em: 11 de fev. 2019.

VIEIRA, H.C.A.; OLIVEIRA, R.R.; SILVA, M.L.; SILVA, D.L.S.; CONCEIÇÃO, G.M.; OLIVEIRA, H.C. Briófitas de ocorrências em São João do Sóter, Maranhão, Brasil. Acta Brasiliensis, v.1, n.2, p.8-12, 2017. Disponível em: <https://doi.org/10.22571/Actabra12201745>. DOI: 10.22571/Actabra12201745.

YANO, O. An additional checklist of Brazilian bryophytes. Journal of the Hattori Botanical Laboratory, v.66, n.4, p.371-434, 1989. Disponível em:<http://dx.doi.org/10.1590/2175-7860201566409>. DOI: 10.1590/21757860201566409.

YANO, O. A new additional annotated ckecklist of Brazilian bryophytes. Journal of the Hattori Botanical Laboratory. v.78, p.137-182, 1995.

YANO, O. Novas ocorrências de Bryophyta para vários estados do Brasil. Acta Amazonica. v. 34, n.4, p.559-576, 2005. Disponivel em: <http://dx.doi.org/10.1590/S0044-59672004000400008>. DOI: 10.1590/S004459672004000400008.

YANO, O. Novas adições ao catálogo de Briófitas Brasileiras. Boletim do Instituto de Botânica. v.17, p.142, 2006. 
YANO, O; BORDIN, J.; PERALTA, D.F. Briófitas dos estados do Ceará, Maranhão, Paraíba, Piauí e Rio Grande do Norte (Brasil). Hoehnea. v. 36, n.3, p.387-415, 2009. Disponível em: <http://dx.doi.org/10.1590/S2236-89062009000300002>. DOI:10.1590/S2236-89062009000300002. 